\title{
Zur Diagnostik der Bronchialdrüsentuberkulose im Kindesalter.
}

\author{
Von
}

\section{Dr. Egon Rach,}

Assistenten der Klinik.

Mit 1 Tafel und 3 Abbildangen im Text.

Dass von verschiedenen Erkrankungen die rechte Lunge häufiger befallen wird als die linke, ist eine Erfahrungstatsache, die schon mehreren Autoren aufgefallen ist. So kommt Ghon bei seinen grundlegenden Untersuchungen über den primären Lungenherd der Kinder zu dem Frgebnis, dass die rechte Lunge mehr primäre Lungenherde aufweist als die linke. Diese Tatsache erscheint ihm vom anatomischen Gesichtspunkte aus verständlich, doch stellt er in Aussicht, sie wegen ihrer Wichtigkeit an anderer Stelle besonders zu besprechen. Zum Teil dürfte wohl diese "grössere pathologische Gefährdung der rechten Lunge" (Sukiennikow) auf ihre Grösse im Verhältnis zur linken zurückzuführen sein. Parallel mit der häüfigeren Erkrankung der rechten Lunge geht auch eine häufigere Beteiligung der rechten Bronchialdrüsen. Dass diese schon im normalen Zustande rechts reichlicher vorhanden sind als links und dass besonders die rechtsseitigen tracheobronchialen Drüsen zahlreicher sind als die linksseitigen wird von Sukiennik ow besonders hervorgehoben.

Er unterscheidet an der Gruppe dieser rechtsseitigen tracheobronchialen Drüsen zwei Anteile, einen oberen, der die kettenartig angeordneten, in dem Spalt zwischen der Trachea und der Wirbelsäule gelegenen Lymphdrüsen umfasst, und einen unteren, in welchem die Lymphdrüsen einen Haufen bilden und einen anatomisch genau begrenzten Raum ausfüllen. 
Die topographische Anatomie dieses Raumes, des "rechten tracheobronchialen Raumes", ist von S u ki ennik ow genau beschriehen worden und soll hier wegen ihrer hervorragenden Wichtigkeit ausfürlich wiedergegeben werden.

„Nachdem die Trachea die obere Brustapertur passiert hat, behält sie die mediane Lage bei, macht aber der Wirbelsäule folgend, eine Krümmung nach hinten und entfernt sich somit in ihrem Laufe nach abwärts mehr und mehr von der Vertikalen. In der Höhe der ersten Rippe vor dem rechten Rande der Trachea fliessen die beiden Venae anonymae zur Vena cava superior zusammen, welche fast vertikal zum Herzen zieht. Der Abstand der Vena cava superior vom unteren Teile der nach binten sich krümmenden Trachea ist gleich der Dicke der rechten Arteria pulmonalis, welche, teilweise vom Perikard eingehüllt, den vorderen Teil des Bodens eines zwischen der Vena cava superior und der Trachea entstandenen Raumes bildet. Den hinteren Teil dieses Bodens stellt der rechte Bronchus dar. Die linke Begrenzung übernimmt im hinteren Teile des Raumes die rechte Seite der Trachea, in seinem vorderen Teile - die aufsteigende Aorta. Von rechts oben wird dieser Raum von dem vorbeiziehenden rechten Mediastinalblatte abgeschlossen; die rechte untere Begrenzung bildet die Vena azygos. Die Arteria anonyma von links und oben und der Nervus vagus dexter von hinten beeinflussen den Raum in der Weise, dass er nach oben sich verjünt und in einen Spalt zwischen der rechten Karotis, der Trachea und Wirbelsäule übergeht.

Wir können nach diesen Verhältnissen den beschriebenen Raum als etwa kolbenförmig bezeichnen, wobei der engere Teil des Kolben nach oben rechts von der Trachea liegt, der mittlere den Angulus tracheobronchialis dexter ausfüllt und der untere geräumigere Teil vor der Trachea und dem rechten Bronchus an der Stelle seines Abganges zu liegen kommt."

Die grosse Wichtigkeit dieses rechts neben der Trachea gelegenen und bis zur Abzweigung des rechten Hauptbronchus herabreichenden Drüsenpakets wird schon von fast allen älteren Autoren, die über die Bronchialtuberkulose schrieben, hervorgehoben.

Bevor wir die verschiedenen diesbezüglichen Bemerkungen rasch durchgehen, soll zuerst die alte, von den Franzosen stammende Nomenklatar und Ein. teilung der Lungendrüsen kurz angeführt werden.

Baréty traf die Einteilung in ganglions sus ou intrabronchiques (in den Teilungswinkeln der Bronchen erster bis vierter Ordnung), in ganglions intertrachéo-bronchiques (im Bifurkationswinkel der Trachea), in prétrachéo bronchiques droites et gauches (in den beiden tracheobronchialen Winkeln) und restrosternales 
(rechts nahe der Vena cava superior). H u tin el nennt die prétrachéo-bronchiques auch ganglions juxta-trachéales.

So betont $\mathrm{K} \ddot{\mathrm{u} s} \mathrm{~s}$ die entschiedene Prädisposition dieser Lymphknoten für die Tuberkulose, er meint, dass sie oft verändert sind, ohne dass dafür der normale Weg der lymphogenen Ausbreitung die Erklärung geben kann. Auch $\mathrm{Hutinel}$ erwähnt, dass „Le groupe juxtatracheal gauche (ou groupe prétrachéo-bronchique gauche de Baréty) est moins riche et moins important, que le groupe droit."

Haushalter und Fruhimholz betonen die besondere Disposition der rechten pretracheo-bronchialen Drüsen.

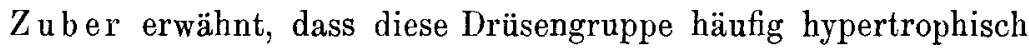
ist und mit der Articulatio sternoclavicularis in Zusammenhang treten kann. An anderer Stelle spricht er von den retrosternalen Drüsen, die rechts eine wichtige Gruppe bilden können, die in Beziehungen mit der Vena cava superior treten kann.

„Parmi les gros vaisseaux, aorte, artères et veines pulmonaires, veine azygos, veine cave supérieure, c'est cette dernière, qui est le plus souvent comprimée grâce à ses rapports immédiats avec la masse des ganglions prétrachéo-bronchiques du côté droit et les ganglions sterno-claviculaires."

Widerh of er gibt eine Zusammenstellung der Fälle von Kompression oder Perforation der Trachea oder der Bronchien durch tuberkulöse Bronchialdrüsen aus dem Kindesalter. Er findet eine Kompression der Trachea in vier Fällen, beider Hauptbronchien in einem, des rechten jedoch in sechs und des linken nur in einem Falle. Perforation der Trachea in einem, beider Hauptbronchien in zweien, des rechten in sieben, des linken hingegen nur in vier Fällen. Es zeigt sich also hier ein weitaus häufigeres Befallenwerden des rechten Bronchus im Vergleich zum linken, also auch ein häufigeres Anschwellen der rechten Drüsen im Vergleich zu den linken. Auch Ghon erwähnt, dass bei Kompression von Bronchien durch vergrösserte käsige Lymphknoten der rechte Hauptbronchus öfter befallen ist als der linke, dass aber hierbei auch Bronchen erster oder zweiter Ordnung betroffen sein können. In 28 Fällen von Einbruch tuberkulöser Lymphknoten in die Bronchen, teils frischeren, teils älteren Datums, ist ebenfalls der rechte Hauptbronchus gegenüber dem linken bevorzugt.

Auch Völker (zitiert bei Ribbert) weist darauf hin, dass die Perforation von Drüsen am häufigsten in den rechten Hauptbronchus erfolge und führt dies darauf zurück, dass hier die Drüsen auch schon in der Norm besonders dicht und fest dem Bronchus anliegen. 
Dass bei der tuberkulösen Erkrarikung der Bronchialdrüsen im Kindesalter dieser rechte tracheobronchiale Raum oft von einem Drüsenpaket ausgefüllt ist, das durch Verschmelzung verkäster Drüsen entstanden ist, und - in seiner Gestalt diesem Raume entsprechend - eine charakteristische Kolben- oder Dattelform zeigt, ist auch mir bei zahlreichen Obduktionen, die ich im Hinblick auf diese Frage mit ansah, aufgefallen.

In einer Arbeit, „Beiträge zur Röntgendiagnostik der Lungentuberkulose im Kindesalter, " habe ich nachgewiesen, dass es mitunter glücken kann, diese Art von Drüsenschwellung radiologisch zu erkennen. Ich habe damals diese Drüsen als paratracheale Drüsen bezeichnet. Unter diesen wäre nach Ghon der obere kettenartige Anteil der tracheobronchialen Drüsen Sukiennikows zu verstehen. Obwohl die beiden Anteile dieser Drüsengruppe zusammenhängen und wenigstens bei Kindern (Ghon) oft ohne scharfe Grenze ineinander übergehen, obwohl daher besonders bei ausgedehnter Verkäsung eine Unterscheidung schwierig sein mag, ist es doch richtiger, sie nicht als paratracheale, sondern als tracheobronchiale, und zwar mit Ghon als obere tracheobronchiale zu bezeichnen, während als untere tracheobronchiale Sukiennik ows Bifurkationsdrüsen zu verstehen sind.

Seit der Beendigung dieser Arbeit habe ich neuerdings eine grosse Anzahl von identischen radiologischen Bildern gesehen und auch in einer weiteren Reihe von Fällen den radiologischen Befund anatomisch bestätigt gefunden. Es besteht eine bemerkenswerte Übereinstimmung des radiologischen Bildes mit der Konfiguration des von Sukiennikow beschriebenen rechten oberen tracheobronchialen Raumes. Der Drüsenschatten hat auf dem Röntgenbild die Form eines rechts neben der Luftröhre und parallel mit dieser ziehenden Streifens, der oben und unten in einem konvexen Kontur sich mit dem Mittelschattenhals vereinigt. Sukiennik ow beschreibt die Gestalt dieses Raumes als kolbenförmig. Am häufigsten zeigt anatomisch und radiologisch das ihn ausfüllende Drüsenpaket Dattelform und es könnte der Schatten demnach am einfachsten als dattelförmiger Paratracheal-Schatten bezeichnet werden.

Ähnliche Befunde verzeichnen auch Keiner, sowie Neuhaus; auch Ribadeau Dumas, Weil und Samet erwähnen in ihrer Mitteilung: „Radiographie et Radiothérapie de l'adénopathie médiastine" als "bande sombre juxtatrachéale" einen ähnlichen Schatten, der, wie überhaupt die meisten, tuberkulösen Drüsen oder Lungenherden entsprechenden Schatten, beim Kinde öfter rechts als links auftritt. 
Jedoch wird eine einseitige radiologische Diagnose dem Kliniker nicht genügen, der seine Aufgabe darin sieht, die mit Hilfe der verschiedenen SpezialUntersuchungen gewonnenen Einzelsymptome zu einem organischen Ganzen innig zu verschmelzen, um ein einheitliches Bild der Krankheit zu gewinnen.

Dementsprechend werden wir uns zu fragen haben, welch andere als radiologische Symptome die Schwellung und Verkäsung der rechten oberen tracheobronchialen Drüsen hervorrufen kann. Dabei werden wir bald zur Überzeugung kommen, dass der Intumeszenz gerade dieser Gruppe der Bronchialdrüsen eine ganz besondere Bedeutung und spezielle Symptomatologie zukommt. Diese beruht auf den von Sukiennikow angegebenen Lagebeziehungen zu den so wichtigen anatomischen Gebilden in ihrer Nachbarschaft, die einer Kompression ausgesetzt sind.

Es lassen sich sogar die klinischen Erscheinungen, die bei der Schwellung dieser Drüsengruppe aufzutreten pflegen, mit den älteren französischen Autoren und mit Wi derh of e $\mathrm{r}$ in verschiedene Gruppen bringen, je nachdem ob Nerven, Gefässe oder die Luftwege in Mitleidenschaft gezogen worden sind. So nennen Barthez und Rilliet unter den durch Druck der Drüsen auf die Nerven hervorgebrachten Symptomen krampfhustenartigen, rauhen, schmerzhaften Husten, asthmatische Anfälle, die meist nachmittags auf ganz unbedeutende Anlässe hin auftreten und zeitweise auftretende Heiserkeit, bei der der Kehlkopf selbst vollkommen gesund ist. Hier sei auch erwähnt, dass B aréty in $26 \%$ obduzierter Fälle verschiedenen Alters mit Bronchialdrüsentuberkulose eine Kompression oder Adhärenz des Vagus und seiner Äste finden konnte. Andererseits soll die Kompression der Vena cava superior, die in Ausnabmsfällen (Tonnelé) bis zur Obliteration dieses Gefässes gehen kann, nach $\mathrm{Zuber}, \mathrm{Hutinel}$, Wider h of er zu intermittierender Zyanose und zu intermittierendem Ödem des Gesichtes, mitunter angeblich auch zu Ödem und zu Hämorrhagien in den Meningen führen können.

Schon Barthez und Rilliet erwähnen ferner ein in der Ferne hörbares Keuchen, das intermittiẹrend auftritt und am ehesten dem Keuchen Kropfkranker zu vergleichen ist. Sie erklären dieses Keuchen durch Druck auf die Trachea und auf die Bronchen.

Nach Widerhofer kommt es bei Stenose der untersten Trachea oder der Hauptbronchen infolge von tuberkulöser Bronchialdrüsenschwellung zu inspiratorischer Dyspnoe, die von einem lauten, weithin hörbaren, tönenden (keuchenden) inspiratorischen Atemgeräusch begleitet wird. 
Sie soll sich von der inspiratorischen Dyspnoe infolge von Larynxstenose bei Krupp besonders dadurch unterscheiden, dass bei dieser der Kopf nach rückwärts gebeugt wird, während bei jener der Kopf nach vorne gebeugt wird, der Larynx kaum nennenswerte Exkursionen während der Respiration macht und die Stimme völig oder fast völlig frei ist.

Ein keuchender Stridor kann bei Säuglingen sogar auffälliger und diagnostisch wertvoller sein als alle übrigen klinischen Symptome der Bronchialdrüsentuberkulose (Dämpfung im Bereich der Region de Mattité ganglionaire antérieure (Guéneau de Mussy), Dämpfung über dem 5.-8. Brustwirbel, Tastbarkeit retrosternaler Drüsen (Fonssagrives), Bronchophonie über dem 1.-4. Brustwirbel beim Schreien, Flüsterstimme daselbst beim leisen Sprechen (signe d'Espine), zeitweise zu Erstickungsanfällen exazerbierende Dyspnoe, Erweiterung der Venen des Gesichtes, Exophthalmus (Friedjung), sowie Veränderung des Stimmtimbres).

Variot und Bruder, Guinon, Bougarel, Schick haben die wichtige Tatsache festgestellt, dass dieser Stridor bei Säuglingen vorwiegend exspiratorisch anftritt.

$\mathrm{Ob}$ das von $\mathrm{W}$ iderh of er bei rachitischen, auf Bronchialdrüsenschwellung verdächtigen Säuglingen geschilderte, laute, weithin vernehmbare "Singen, Gimmen, Rasseln", mit diesem Phänomen identisch ist oder nicht, kann nicht entschieden werden, jedenfalls weist die Widerhof er sche Darstellung nicht alle charakteristischen Merkmale desselben auf. Es wird nur angegeben, dass es sich im höchsten Grade so anhört, als würde eine Flüssigkeitssäule auf- und absteigen, dass es an der Trachea leicht mit der Hand zu fühlen ist und dass dabei weder das In- noch das Exspirium wesentlich erschwert ist, nur mässig frequentere Atmung besteht.

Die erste einwandfreie Beobachtung stammt von Variot und Bruder (Demonstration in der Sitzung vom 23. II. 1904 der Sociéte de pédiatrié zu Paris). Ein 6 Monate altes Mädchen leidet seit 2 Monaten an Hustenanfällen, die auftreten, sobald man das Kind berührt. Seit der gleichen Zeit exspiratorisches Keuchen von scharfem Klang, das auch während des Saugens anbält, aber mitunter im Schlaf aufhört. Der Thorax stark gewölbt; links hypersonorer Scball; keine Einziehungen; keine Erstickungsanfälle. Die Intubation ändert nichts an der Intensität des Geräusches. Daraus wird geschlossen, dass das Geräusch in der Trachea oder in den Bronchien entsteht. Kehlkopfspiegelbefund negativ, keine Erschwerang der Nasendurchgängigkeit wio bei Adenoiden, daher die Bezeichnung cornage exspiratoire bronchitique. Zum Unterschied davon sei der kongenitale stridor immer inspiratorisch, beginne in den ersten Tagen nach der Geburt und verschwinde nach 1 oder 2 Jahren. Anatomisch finde sich beim kongenitalen Stridor eine Missbildung des Kehlkopfes. In 2 Fällen wurde von Variot Aufhören des Geräusches während der 'Tubage beobachtet. 
In der Diskussion meint $\mathrm{Guin}$ on, dass eine Bronchialdrüsenschwellung nicht von der Hand gewiesen werden kann.

Später berichtete Variot, dass die Röntgenuntersuchung des am 23. Febr. vorgestellten Kindes rechts etwas oberbalb des Herzschattens einen der Gegend des rechten Hilus entsprechenden Schatten zeigte, dor vermutlich einem den rechten Hauptbronchas komprimierenden Drüsenpaket entsprach.

Seither wurden 2 weitere Fälle mit ähnlichem Keachen beobachtet. 1. Ein $4 \%$ Monate altes Mädchen, das mit 3 Monaten an Bronchitis erkrankte. Das Kind schreit mit klarer Stimme, dabei verschwindet das Keuchen; radiologisch links oberbalb des Herzens ein bis zum Schlüsselbein reichender Schatten. Der Herzschatten scheint verbreitert.

2. Ein 6 Monate aiter Knabe, der seit 3 Monaten hartnäckigen Husten, häufiges Erbrechen, plötzliches Erwachen des Nachts mit geräuschvoller Atmung zeigt. Ebenso wie im früheren Fall ist die Inspiration geräuschlos. Ausgebreiteter Schatten links oberhalb des Herzens. Auch hier wird Bronchostenose infolge einer Drüsengeschwulst vermutet. Anskultation und Perkussion negativ. Dass das Keuchen exspiratorisch auftrat, wird folgendermassen erklärt: vielleicht komprimiert die Drüsenmasse während der exspiratorischen Verengerung des Brustkorbes stärker den Bronchus als während der Inspiration, wo die Drüsen infolge der Thoraxerweiterung in zentrifugaler Richtung gezogen werden.

Guinon berichtet über 2 Beobachtungen:

1. Fall: Betrifft einen von einer phthisischen Mutter stammenden Knaben, der schon in der 3. Lebenswoche Kurzatmigkeit zejgte. Mit 3 Monaten Beschleunigung der Atmung, pfeifender exspiratorischer Stridor, der während des Schlafes verschwindet. Seltener, ebenfalls pfeifender Husten. Geschrei schwach, ohne Besonderheiten. Perkussion negativ, Auskultation wegen des trachealen Stridors fast nnmöglich. Mit 4 Monaten der Husten viel stärker, fett, stridorös, ohne Paroxysmen, erweckt das Kind in der Nacht. Die Dyspnoe ist stark und wird von rhythmischen Bewegungen des Kopfes und von einer tiefen Einziehung rings um den unteren Anteil des Brustkorbes begleitet. Die Dyspnoe ist exspiratorisch wie bei kapillärer Bronchitis. Tod durch Erstickung mit 6 Monaten. Autopsie: Taberkulose der supra. und infraklavikularen Drüsen sowie der trachealen und bronchialen Drüsen mit leichter Kompression der Trachealwand, sowie der unteren und inneren Wand des linken Hauptbronchus.

Fall 2: Beginn der Erkrankung bei einem dreimonatigen Brtstkind einer tuberkulösen Frau. Mit 4 Monaten erscheint die Exspiration von einem Pfeifen oder besser von einem geringen Rasseln begleitet, das von den Schwingungen kleiver, in der Trachea gebildeter Bläschen herzurühren scheint. Stimme nicht. beteiligt, das Geschrei laut und scharf. Kreisförmige inspiratorische Einziehung der unteren Thoraxpartien, vom Sternum aus die falschen Rippen ergreifend. Tod unter Krämpfen an Erstickung. Die Obduktion ergibt ausser einem Taberkel im Pedunculus enorme tuberkulöse Drüsen. Unregelmässig rundliche, walnussgrosse Drüsengeschwulst, rechts und oberhalb des Arcus aortae, unter der Arteria anonyma und hinter der Vena anonyma, die abgeplattet erscheint. Rückwärts wird die vordere Trachealwand komprimiert. Links findet sich eine Drüsengeschwulst, die nur ein Drittel der Grösse der rechten erreicht und keine mechanische Rolle gespielt za baben scheint. Unter der Bifurkation eine ebenfalls nussgrosse Drüsengeschwulst, die besonders den rechten Hauptbronchus zur Seite drängt. Alle Drüsen sind verkäst und erweicht. 
Epikrise: Das erste Symptom war eine Art von Keuchen, das die Eltern zunächst einem Stockschnupfen zuschrieben, das aber bald das Krankheitsbild beherrschte. In einem der Fälle Guin ons verminderte sich das Keuchen, wenn man das Kind nach vorne neigte. Der Husten ist pfeifend, ohne jede Heiserkeit oder Erstickung. Die Einziehungen sind auf den ersten Blick sehr auffallend, sie treten gleichzeitig über und unter dem Sternum auf und bilden eine Furche im Niveau der faschen Rippen.

Differentialdiagnose: Gegen Fremdkörper spricht der progressive Charakter und die geringere Intensität der Dyspnoe. Adenoide hindern beim Trinken und rufen eine geräuschvolleres Keuchen hervor, das durch Zuhalten der Nase unterdrückt werden kann. Die Larynxstenose gibt ein kruppartiges, heiseres Geräusch (wie bei stridulöser Laryngitis) oder Aphonie. Bei kongenitalem Stridor besteht ein sonorer inspiratorischer Stridor ohne Dyspnoe. Die Stenose infolge von Thymushypertrophie zeigt ein sehr veränderliches Krankheitsbild. Auch die exspiratorische Dyspnoe der kapillären Bronchitis oder der Bronchopneumonie ist mitunter von einem trachealen Pfeifen begleitet, das aber durch Husten zeitweise verändert oder unterdrückt wird.

Bougarel kommt am Schlusse seiner ausführlichen Untersuchungen zu folgenden Ergebnissen:

1. Die Schwellung der tracheobronchialen Drïsen kann bei Säuglingen als erstes klinisches Symptom ein charakteristisches Keuchen „cornage exspiratoire" hervorbringen, das mit dem zweiten bis sechsten Monat beginnt.

2. Es ist ein regelmässiges lautes, auf Distanz hörbares Geräusch von feuchtem Charakter, das besonders exspiratorisch auftritt, während die Inspiration fast geräuschlos ist.

Es erinnert an ein kurzdauerndes Schnurren oder an das Schnarchen im Schlafe; es bleibt sich stets gleich und hat einen hohen, mitunter sogar scharfen Klang. Von anderen Stridorarten, mit denen es diesen Klang gemeinsam hat, unterscheidet sich das Keuchen der Bronchialdrüsentuberkulose durch seinen feuchten Charakter. Es hört sich an, als würde das Kind den Buchstaben $R$ aussprechen. Dabei scheinen die Luftwege von Schleim erfüllt zu sein, der durch den Luftstrom in Schwingung gerät. Das ganz eigentümliche Geräusch war in drei Fällen durch den ganzen Saal hindurch zu hören und übertönte alles übrige.

3. Die Kinder bleiben dabei lustig, die Krankheit entwickelt sich langsam im Laufe mehrerer Monate, bis eines Tages Komplikationen (Erstickung oder Hirnhautentzündung) plötzlich sich einstellen.

4. Es zeigt im allgemeinen zunehmenden Charakter, kann aber durch Sohrumpfung oder Entleerung des Drüseninhaltes ausheilen. Die Prognose ist in allen Fällen ernst.

5. Das exspiratorische Keuchen scheint darauf zu beruhen, dass im Moment der Exspiration durch die Elastizität der Lungen auf die 
Drüsen ein Druck ausgeübt wird, der das Kaliber der im Säuglingsalter biegsamen Luftwege verengt. Später als im 3. Lebensjahr begegnet man dem Keuchen nicht mehr, da zu dieser Zeit die Trachea und die Bronchien ihre definitive Gestalt und eine festere Konsistenz haben.

6. In 10 Beobachtungen, von denen 5 durch die Röntgenuntersuchung und 2 durch die Autopsie kontrolliert wurden, unterschied der exspiratorische Charakter, der schliessliche Ausgang und die progressive Zunahme des Geräusches das Keuchen vom kongenitalen Stridor und vom Thymuskeuchen, die beide inspiratorisch sind, frühzeitig beginnen und später schwächer werden, um am Ende des ersten Lebensjahres aufzuhören. Der heimtückische Verlauf ohne dramatische $Z$ wischenfälle unterscheidet das exspiratorische Keuchen von dem durch laryngeale Krisen unterbrochenen inspiratorischen Keuchen, das rasch sich entwickelnde Drüsenschwellungen begleitet (die nicht tuberkulösen Drüsenschwellungen von Simon und Greffrier und die tuberkulösen Drüsenschwellungen von Ley, Rilliet und Barthez).

7. Die gewöhnlichen physikalischen Untersuchungsmethoden, besonders die Auskultation sind beim Säugling schwer verwendbar, darum muss die Radioskopie die Diagnose entscheiden. Man sieht auf dem Leuchtschirm rechts oder links vom Mittelschatten über dem Herzen ausgedehnte, geschwollenen Drüsen entsprechende Schatten, mit rundlichen Konturen, ohne Pulsation. Abgesackte Empyeme zeigen eine längliche Form und sehr spitze Winkel. Der Röntgenbefund war in allen Fällen positiv.

Die anatomischen Befunde, die sich mit den radioskopischen decken, sind bei dem Keuchen infolge von Drüsenschwellung folgende:

Umfangreiche Drüsen umgeben rom Sternoklavikular-Gelenk die Trachea bis hinab zur Bifurkation, rechts ebenso wie links, dabei kann sich die Geschwulst bis über das Niveau des Aortenbogens hinaus erstrecken, nach rückwärts bis über die Vena anonyma hinaus. Sie kann sagar die Klavikula überschreiten und die Halsdrüsen in Mitleidenschaft ziehen. Ihre Anordnung zur Trachea ist eine derartige, wie sie Baréty beschrieben hat. Es handelt sich nicht um zirkuläre Verengungen, sondern um seitliche Kompressionen, die durch eine oder mehrere der Trachea anhaftende Drüsen ausgeübt wird. Daraus folgt eine mehr oder weniger ausgebreitete Abplattung der Bronchien. Die Lungen zeigen Emphysem, der Larynx zeigt normale Beschaffenheit.

Schick kommt auf Grund von 36 Eigenbeobachtungen, die grösstenteils durch die Pirquetsehe Kutanreaktion und durch die 
Röntgenuntersuchung (Slukas), zum Teil auch durch die Sektion überprüft werden konnten, zu folgenden Resultaten: Das Keuchen ist in der ganzen Zeit des Exspiriums in Ruhe des Kindes meist weithin hörbar. Es klingt am ähnlichsten dem Geräusch bei Asthma bronchiale und kapillärer Bronchitis. Das Exspirium erscheint deutlich verlängert und angestrengt, das Inspirium ist kaum hörbar. Wenn dieses Symptom einmal aufgetreten ist, so verschwindet es nicht so bald und es kommt unter dem Einfluss der dauernd erschwerten Exspiration zur Ausbildung von Lungenblähung mit Tiefstand der unteren Lungengrenzen und Schachtelton über den geblähten Lungenpartien.

Die anatomische Grundlage für das Zustandekommen des Symptoms bildet nach den meisten Sektionsbefunden (in einigen Fällen war keine bestimmte Ursache nachweisbar) die Kompression eines Hauptbronchus, meist des rechten, durch eine verkäste und vergrösserte Lymphdrüse. Die Stelle der Kompression befindet sich in der kurzen Strecke zwischen Bifurkation und Abgangsstelle des Bronchus für den rechten bzw. linken Oberlappen. Schick erklärt das exspiratorische Auftreten des Keuchens damit, dass eine geringfügige Stenose im Inspirium, wo das Lumen des Bronchialbaumes entfaltet ist, noch keine Erscheinungen macht, während solche im Exspirium bereits bestehen. Die Stenosierung wird um so leichter bemerkbar, je kleiner das Lumen des stenosierenden Rohres ist; daher entsteht die rein exspiratorische Atemerschwerung am leichtesten im ersten Lebensjahre.

Bei Kindern jenseits des ersten Lebensjahres ist das exspiratorische Keuchen wohl auch durch tuberkulöse Veränderungen bedingt, doch ist die anatomische Grundlage des Phänomens nicht mehr oinheitlich.

Der diagnostische Wert dieses Symptoms liegt nach Schick besonders darin, dass das exspiratorische Keuchen als ein par distance wahrnehmbares Zeichen die diagnostischen Erwägungen rasch in das richtige Fahrwasser lenkt.

Ausserdem wäre noch hervorzuheben, dass auch die ganz eigentümliche Beschaffenheit des Hustens ein höchst wertvolles Symptom darstellt, da auch der Husten auf Distanz hörbar ist und auf jeden, der ihn in typischer Weise einmal gehört und seine Eigentümlichkeit erfasst hat, einen unvergesslichen Eindruck macht. Er wird von Fried jung als „keuchend, pfeifend, von auffallend grosser Tonhöhe", von anderen Autoren als "hoch, fast metallisch klingend" geschildert. Ich möchte anf Grund meiner Beobachtungen den Husten als kreischend oder als schrill bezeichnen, da seine Klangfarbe durch hohe 
metallische Obertöne charakterisiert ist und an das Tönen eines gesprungenen Glases erinnert.

Alle diese Symptome beweisen somit nicht nur die Anwesenheit von geschwellten Bronchialdrüsen, sondern ausserdem noch die Kompression der Nachbarorgane durch dieselben.

Ich verfüge über zwei anatomische Präparate, die in toto gehärteten Thoraxorgane von Kindern, die das Symptom des exspiratorischen Keuchens geboten hatten. In beiden Fällen hatte die Vergrösserung der rechten oberen tracheobronchialen Drüsen zur Verengerung des rechten Hauptbronchus geführt, wobei in beiden Fällen am unteren Pol dieses Drüsenpaketes ein Einbruch in die vordere Wand desselben erfolgte. Es sei mir gestattet, eine kurze anatomische Skizzierung dieser beiden Fälle bier einzuschalten.

Anna B., P.Nr. 33, vom 16. XII. 1911. Im. Alter von 6 Monaten in die Klinik aufgenommen. Mutter leidet an offener Lungentuberkulose. Das Gesicht bleich, die Venen am Nasenrücken stark ausgedehnt. Papulonekrotische Tuberkulide am Stamm und an den Gliedern. Die Atmung stark beschleunigt, leichte Zyanose der Hände und Füsse, anhaltendes, auf Distanz hörbares lautes, eigentiimıliches exspiratorisches Keuchen. Sehr häufig eigentümlich klanghaltiger und schriller Husten, der in seiner Klangfarbe etwas an den Ton eines gesprungenen Glases erinnert. Über dem rechten Oberlappen Dämpfung, bronchiales In-und Exspirium und klingendes Rasseln. Die Lumbalpunktionsflüssigkeit leicht getrübt; Tuberkelbazillen darin mikroskopisch nachweisbar; zeitweise Bewusstlosigkeit, klonische Krämpfe der rechten Körperbälfte. Die Röntgenaufnahme (s. Tafel I Abb. 1) zeigt einen dichten, gleichmässigen Schatten, der mit Ausnahme der Spitze den ganzen rechten Oberlappen einnahm, medialwärts mit dem Mittelschattenhals konfluierte und sich der Lappengrenze entsprechend unten, mit einer schräg von oben und aussen nach innen und unten abfallenden scharfen Linie begrenzte. Im Bereich desselben scharf umschriebene ausgedehnte Aufhellung mit gebuchtetem Kontur. Ausserdem zahlreiche, über beide Lungen gleichmässig verstreute, ungefähr hanfkorugrosse Schattenflecke, die Lungenränder tiefstehend, die Herzspitze freiliegend. Obduktion (Professor Oskar Störk): Meningitis tuberculosa mit Bildung eines sehr reichlichen, sulzigen Exsudats an der Basis. Miliartuberkulose des grossen Kreislaufes (Leber, Milz und Nieren); vereinzelte tnberkulöse Geschwüre im Cökum und Colon aszendens; chronische käsige Tuberkulose der mesenterialen Lymphdrüsen. Ausgedehnte Verkäsung der tracheobronchialen Lymphdrüsen mit Durchbruch der vorderen Wand des rechten Stammbronchus knapp an der Bifurkation und beträchtlicher Stenose des Bronchiallumens. Ausgedehte Verkäsung im Bereiche des rechten Oberlappens mit Bildung einer ausgedehnten zentralen Zerfallshöhle, die bis ungefähr $5 \mathrm{~mm}$ an die laterale Oberfläche der rechten Lunge heranreicht. Subakute disseminierte Tuberkulose aller Lungenabschnitte in Form etwa hanfkorngrosser, ziemlich dicht und gleichmässig ausgesäter verkäsender Knötchen.

Die in situ mit Formolalkohol gehärteten Brustorgane wurden mir in dankenswerter Weise von Herrn Professor Störk uberlassen. Sie waren durch transversale Schnitte in Scheiben zerlegt. Ein knapp über der Bifurkation angelegter Schnitt (s. Figur 1) zeigt ein in sagittaler Richtung $25 \mathrm{~mm}$ messendes unregel- 
mässig gestaltetes, gleichmässig verkästes Drüsenpaket, das mit dem hintersten Anteil seiner medialen Fläche sich an die rechte vordere und rechte seitliche Fläche der Trachea anlebnt. Diess erscheint hierdurch im mässigen Grade zu einem von links und vorn nach rechts und hinten gerichteten Spalt abgeplattet. Lateralwärts springt diese Geschwulst gegen die Lunge vor, von der sie durch die Vena azygos geschieden ist. An ihrer vorderen Fläche verläuft die Vena cava superior, die gerade an der Einmündung der Azygos getroffen ist. An ihrer medialen Fläche ist der Arcus aortae getroffen. Nach oben zu verschmälert sich die Geschwulst, um ungefähr im Niveau der rechten Lungenspitze einen bohnengrossen Querschnitt zu zeigen. An dieser Stelle liegt sie hinten der rechten Fläche der Trachea an, die eiuen normalkonfigurierten Querschnitt zeigt. An

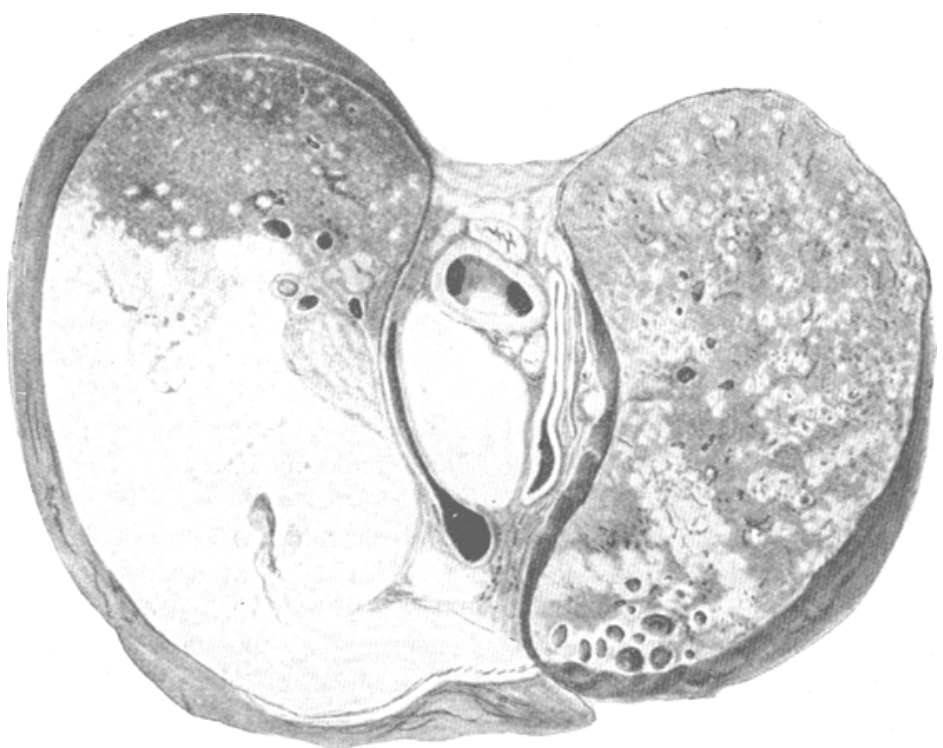

Fig. 1 .

ihrer vorderen Fläche verläuft die rechte Karotis. Auch nach unten zu verschmälert sich das Drüsenpaket. Es legt sich im Niveau der Bifurkation der vorderen Wand des rechten Hauptbronchus an und verschmilat etwas weiter unten ohne eine scharfe Grenze mit den ebenfalls mächtig vergrösserten und gleichmässig verkästen im Bifurkationswinkel gelegenen Drüsen. Hierdurch erscheint der rechte Hauptbronchus sowohl in seiner oberen, als auch in seiner vorderen und unteren Zirkumferenz in diese Drüsen wie eingemanert und auf dem Horizontalschnitt zu einem von lateral und vorn nach medial und hinten gerichtetem Spalt abgeplattet. Auch der linke Hauptbronchus scheint, zwar in geringerem Grade, aber doch ebenfalls abgeplattet. Seiner topographischen Lage nach entsprach somit dieses Drüsenpaket völlig dem oben charakterisierten rechten tracheobronchialem Raum von Sakiennikow.

Alfred L., gestorben im Alter von 7 Monaten unter dem klinischen Zeichen von expiratoriscfer Dyspnoe und Meningitis tuherculosa, Die Obdaktion (Prof. Anton Ghon) ergab chronische Tuberkulose der Hals-, Lungen- und Bronchial- 
drüsen, Kompression des rechten Hauptbronchus durch tracheale Drüsen und solche der Bifurkation mit Darchbruch einer verkästen Drüse in denselben.

An den in toto nach $\mathrm{K}$ a y serling konservierten Thoraxorganen (Museumspräparat der Klinik Nr. 708) ist die hintere Wand der Trachea und der Bronchien samt ihren grösseren Stämmen von hinten aufgeschnitten. Man übersieht so ein knapp unter der Bifurkation an der vorderen Wand des rechten Hauptbronchus befindliches Geschwür mit zackigen Rändern. Dieses führt in das Innere eines ungefährt dattelgrossen und dattelförmigen Drüsenpakets, das sich rechts neben der Trachea nach aufwärts erstreckt und im Bereich der Bifurkation der rechten vorderen Zirkumferenz der Trachea und der vorderen Wand des rechten Hauptbronchus angelagert erscheint. Im Bifurkationswinkel ein kastaniengrosses, von vorne nach rückwärts abgeplattetes, gleichmässig verkästes Drüsenpaket, das bei der Kompression des Bronchus gleichsam als Widerlager diente. Im rechten Lungenoberlappen eine bohnengrosse Kaverne, über beide Lungen gleichmässig verteilt zahlreiche bis hanfkorngrosse, verkäste Tuberkel.

Andererseits konnte ich eine Anzahl von Säuglingen mit den beiden Kardinalsymptomen intumeszierender Bronchialdrüsentuberkulose, mit exspiratorischem Keuchen und schrillem Husten beobachten, die den charakteristischen dattelförmigen Paratrachealschatten zeigten.

Als Paradigma sei einer dieser Fälle ausführlich wiedergegeben:

Als Leopold D. (P.-Nr. 535 v. 18. VI. 1912) 5 $1 / 2$ Monate alt war, starb seine $7 \frac{1}{2}$ jährige Schwester an Lungentuberkulose. Mit 7 Monaten zeigte er positive Kutanreaktion und wurde mit 13 Monaten wegen Röchelns adenotomiert. Dieses Röcheln oder Keuchen wurde durch diese Operation nicht gebessert, sondern

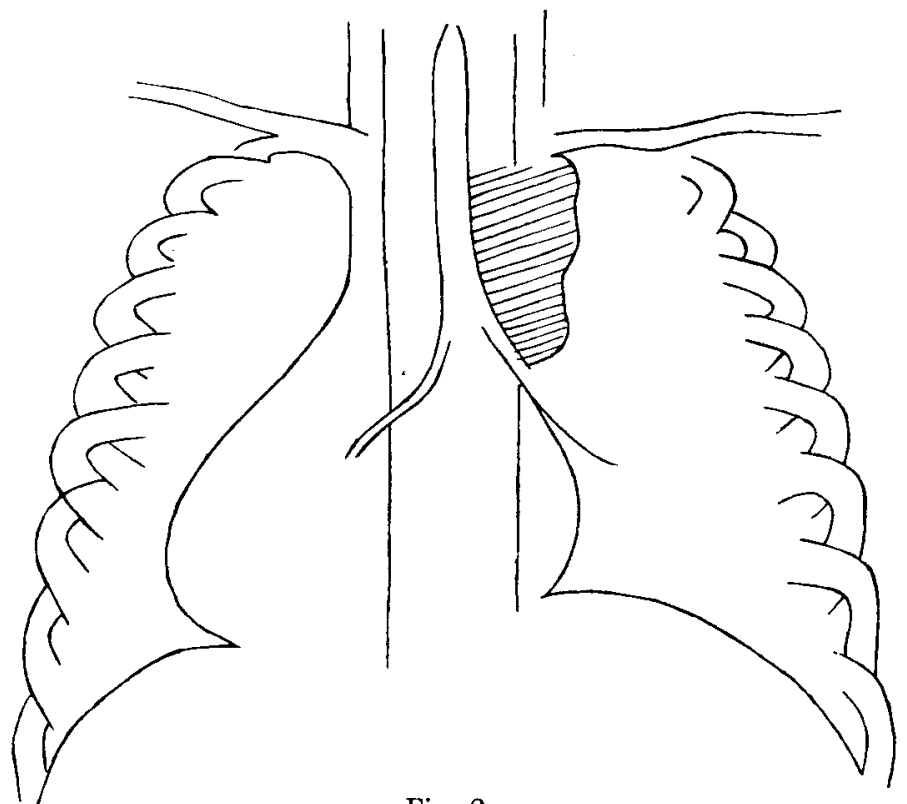

Fig. 2.

Pause einer ventrodorsalen Einzelschlagaufnahme von Leopold D. 
hielt in wechselnder Stärke an. Seit der gleichen Zeit Husten, der ähnlich wie Keuchhusten klingt. Aufnahme in die Klinik im Alter von 18 Monaten. Zu dieser Zeit über dem rechten Oberlappen vorne und rückwärts Schallverkürzung. Atmung beschleunigt, das Atemgeräusch übertönt von einem lauten expiratorisch stärker als inspiratorisch hörbarem Stridor. Die Röntgenaufnabme (s. Figur 2), die mehrmals vorgenommen wurde, zeigte jedesmal einen Schatten am rechten Hilus, der gegen das Lungenfeld allmählich abnahm und etwas verwaschen war. Ausserdem aber eine gegen das Lungenfeld scharf abgesetzte Verbreiterung des Mittelschattenhalses, deren laterale Grenze in Form einer höckerigen Linie parallel der Trachea verlief und sich in einem nach unten konvexen Bogen im Niveau der Bifurkation mit dem Herzschatten vereinigte. Von sonstigen klinischen Symptomen wäre zu erwähnen, dass zeitweise starke Zyanose bestand, eine Andeutung von Trommeluschlägelfingern wahrnehmbar war, dass ferrer die HantVenen vorne oben auf der Brust erweitert waren und dass der Husten eigentümlich schrill und metallisch klang. Das Kind wurde nach mehrmaliger Bestrah. lung, wobei im ganzen auf Brust und Rücken je $4 \mathrm{Hol}$ k knechtsche Einheiten gegeben wurden, gebessert entlassen und im Alter von 3 Jahren nachuntersucht. Damals zeigte es guten Ernährungszustand,'keine Zeichen von seiten des Respirationsapparates und einen nicht wesentlich geänderten radiologischen Thoraxbefund.

Auf Grund dieser angeführten Beobachtungen soll selbstrerständlich durchaus nicht behauptet werden, dass der rechtsseitige dattelförmige Paratrachealschatten in der Weise für das exspiratorische Keuchen charakteristisch ist, dass er für die Diagnose direkt erforderlich wäre. Falls aber ein solcher Schatten sich bei einem Kinde mit positiver Kutanreaktion und exspiratorischem Keuchen findet, ist es naheliegend, einen Zusammenhang zwischen diesen klinischen und jenen radiologischen Symptomen anzunehmen, kann ein derartiger radiologischer Befund ein wertvolles, ergänzendes Symptom darstellen. Dieser radiologische Befund dürfte auch deswegen häufiger sein, weil die rechtsseitigen Drüsen wegen der Beschaffenheit des Herzschattens und wegen der leichten Abweichung des Trachealbaumes nach rechts der Röntgenuntersuchung besonders zugänglich sind.

Daneben können auch andere radiologische Befunde beim exspiratorischen Keuchen sich finden und die gleiche diagnostische Wertigkeit beanspruchen.

So hat Sl uk a auf den radiologischen Nachweis von Verdichtungsherden in der rechten Lunge, besonders in deren Oberlappen, beim exspiratorischen Keuchen hingewiesen, die auf eine Kompression des rechten Hauptbronchus durch die regionären vergrösserten Drüsen schliessen liessen. Allerdings kann ein derartiger Befund eben nur auf indirektem Wege zur genaueren Aufklärung der Pathologie dieses Symptoms beitragen, doch wird dies in solchen Fällen genügen müssen, in denen bei ausgedehnter bis an den Mittelschatten heranreichender Schattenbildung im Bereich der Lungen eine Differenzierung des 
Drüsenschattens vom Herdschatten nicht möglich ist. In diese Kategorie gehört das Röntgenbild des oben beschriebenen und abgebildeten anatomisch untersuchten Falles der Anna B.

Wie ich oben erwähnte, betont schon Variot, ferner auch Bougarel, sowie Schick, dass nur bei Säuglingen die Kompression eines Hauptbronchus das exspiratorische Keuchen hervorruft, und zwar vermutlich wegen der physiologischen Enge der Trachea und ungenügender Entwickelung ihres elastischen Gewebes im frühesten Kindesalter. Bei älteren Kindern können dagegen andere Symptome als Zeichen einer Kompression von Nachbarorganen, z. B. der keuchhustenartige, quälende Reizhusten (Baretys Coqueluchoide), als Zeichen einer Kompression von Nerven (Rilliet und Barthez) auftreten. Der schrille Husten scheint hingegen beiden Lebensperioden gemeinsam zu sein.

Der keuchhustenartige Husten wird von Widerhofer folgendermassen charakterisiert:

„Dieselben rasch aufeinanderfolgenden kurzen Exspirationsstösse mit allerdings geringeren Stauungserschèinungen im Gesicht - die Reprise meist nur angedeutet oder doch sehr schwach --, nicht gar zu selten am Ende das Erbrechen von Schleim sind die Ähnlichkeits- und zugleich die Unterscheidungsmerkmale von Pertussis. Die Anfälle zeigen auch nicht dieselbe Regelmässigkeit in der Wiederkehr wie die Pertussis, es sind nur solche pertussisähnliche unter den einfach katarrhalischen Husten eingestreut. Es fehlt ihnen auch der typische Verlauf."

Auch einen derartigen Fall mit positiver Kutanreaktion und pertussisartigem Reizhusten konnte ich beobachten, bei dem das
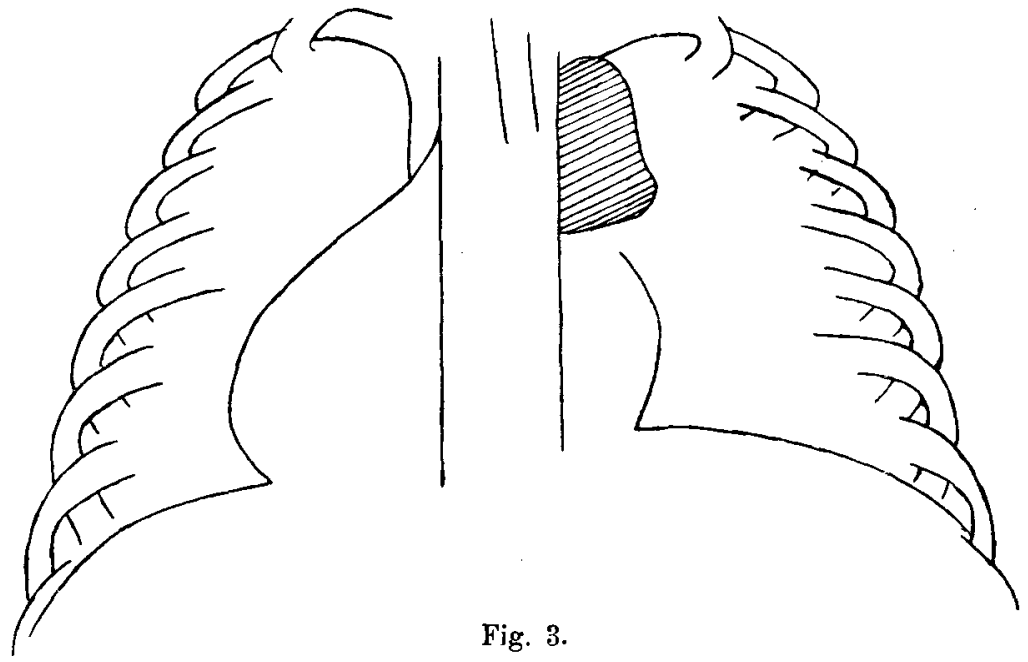

Fig. 3.

Pause einer ventrodorsalen Einzelschlagaufnahme des Gustav S. 
Röntgenbild einen typischen dattelförmigen paratrachealen Schatten auf der rechten Seite zeigte.

Gustav S., 3 Jahre alt, wurde mit einer Pneumonie des rechten Unterlappens aufgenommen. Die Pirquetsche Kutanreaktion war positiv. Nach wenigen Tagen hellte sich die Schallverkürzung und die radiologisch sichtbare Schattenbildung im Bereich des rechten Unterlappens auf, es bestanden jedoch anhaltend über beiden Lungen reichlich zähe Rasselgeräusche und ein hartnäckiger, anfallsweiser Husten von eigentümlich klingender, schriller, kreischender Beschaffenheit, der das Kind bis zu $20 \mathrm{mal}$ in 24 Stunden befiel und sehr belästigte. Dieser Huzten unterschied sich jedoch durch seinen Verlauf - gleich za Beginn war die Zahl der Anfälle am grössten, um später allmählich abzunehmen, und nur anfangs trat hin und wieder stärkere Zyanose und ganz vereinzeltes Erbrechen auf -, sowie durch den Mangel von Reprisen und von typischen krähenden Einziehungen vom echten Keuchhusten. Auch hier fand sich (s. Figur 3) eine Verbreiterung des Mittelschattens nach rechts, die lateral gegen das Lungenfeld in Form einer höckerigen Grenzlinie scharf sich absetzte und die in eịnem nach unten konvexen Bogen im Niveau der Bifurkation mit dem Mittelschatten sich vereinigte.

Ebenso wie in den oben erwähnten Fällen durch das exspiratorische Keuchen wurde in diesem Falle durch den pertussisartigen Husten der radiologische Befund gestützt und gesichert und durch dieses Zusammentreffen von radiologischen und anderen klinischen Symptomen die Diagnose einer bestimmten Form der Bronchialdrüsentuberkulose, nämlich der mit erheblicher Anschwellung einhergehenden und zur Kompression der Nachbarorgane führenden, „intumeszierenden" Form ermöglicht.

\section{Zusammenfàssung.}

Die im rechten tracheobronchialen Raum Sukiennikows gelegenen Drüsen bilden, wenn sie tuberkulös erkranken, mitunter ein diesem Raum sich anpassendes, dattel- oder kolbenförmiges, verkästes Gebilde. Es kann gelingen, dieses radiologisch an einer entsprechenden, der Trachea parallelen, annähernd dattelförmigen Ausbuchtung des Mittelschattenhalses zu erkennen.

Infolge ihrer topographischen Lagebeziehungen können diese Drüsen durch Kompression von Nachbarorganen ganz bestimmte klinische Symptome, im Säuglingsalter durch Kompression des rechten Hauptbronchus einen keuchenden exspiratorischen Stridor (Cornage exspiratoire der Franzosen), später pertussisähnlichen Reizhusten hervorrufen. Diese Symptome können bei positiver Kutanreaktion im Verein mit dem entsprechenden radiologischen Befund die Diagnose einer intumeszierenden (mit beträchtlicher Anschwellung einhergehenden) Bronchialdrüsentuberkulose gestatten. 


\section{Tafe1erklärung.}

Abbildung 1. Ventrodorsales Thoraxradiogramm (Einzelschlagaufnahme) eines 2 jährigen Knaben mit exspiratorischem Keuchen und schrillem, klingendem Husten. Ausser der Ausbuchtung des Mittelschattenhalses, die den rechten oberen tracheobronchialen Drüsen entspricht, ein ins Lungenfeld ausstrahlender Schatten am rechten Hilus und ein dem oberen Rand des rechten Mittellappens entsprechender Schattenstreif.

Abbildung 2. Erklärung im Text S. 11; vgl. Textfigur 1 (anatomisches Präparat des Falles).

\section{Literatur.}

Baréty, A., De l'adenopathie trachéobronchique en général et en particulier dans la scrophule et la phthisie pulmonaire. Thèse, Paris 1874.

Bougarel, L., L'adénopathie trachéo bronchique des nourissons son diagnostic par le cornage bronchitique exspiratoire et la radioscopie. 'Thèse, Paris 1907.

Friedjung, J. K., Beiträge zur Diagnostik und Therapie der Stenosen der oberen Luftwege. Arch. f. Kinderheilk. 1903. XXXV. S. 353.

Ghon, A., Der primäre Lungenherd bei der Tuberkulose der Kinder. Berlin-Wien. 1912.

Guéneau de Mussy, Etade sur l'adénopathie bronchique. Gaz. hebdomadaire 1871. p. 29.

Guinon, M. L., Sur l'adénopathie trachéo-bronchique tubercaleuse des jeunes nourissons. Bull. de la société de pédiatrie 1904. p. 322.

Haugalter et Fruhimbolz, Bull. de la soc. d. péd. 1901.

Hutinel, V., Les maladies des enfants. Paris 1909. T. 1V.

Kein er, Zur Röntgendiagnostik der kindlichen Bronchialdrúsentuberkulose. Verh. der Ges. f. Kinderheilk. 1912. S. 223.

Küss, G., De l'hérédité parasitaire de la tuherculose humaine. Paris 1898.

Neuhaus, H., Beitrag zur Röntgendiagnostik der kindlichen Bronchialdrüsentuberkulose. Fortschritte a. d. G. d. Röntgenstrahlen XX. Heft 4.

Rach, E., Beiträge zur Röntgendiagnostik der Lungentuberkulose im Kindesalter. Zeitschrift f. Kinderheilkunde VIII. Heft 4, 1913.

Ribadeau-Dumas, W eil et Samet, Radiographie et radiothérapie de l'adénopathie médiastine. Bull. de la soc. de péd. de Paris. I. 15. Nr. 8. p. 442 à 454.1913

Rilliet et Barthez, Traité clinique des mal. de l'enfance.

Schick, B., Expiratorisches Keuchen als Symytom der Lungendrüsentuberkulose im ersten Lebensjahre. Wiener klin. Wochenschrift 1910. Nr. 5.

Sluka, E., Übor Röntgenbefunde bei tuberkulösen Kindern mit exspiratorischem Keuchen. Wiener klin. Wochenschrift 1910. S. 156. 
Sukiennikow, W., Topographische Anatomie der bronchialen und trachealen

Lymphdrüsen. Berliner klin. Wochenschrift 1913.

Tonnelé, zitiert nach $\mathrm{Hu}$ tinel.

Variot et Bruder, Bull. d. 1. Soc. d. péd. 1904. p. 51. Séance du 23. II.

Vari ot. Deux nouveaux cas de cornage bronchitique exspiratoire chez d. nourissons. Bull. de l. Soc. de péd. 1904.

Widerhofer, H, Die Erkrankungen der Bronchialdrüsen in Gerhards Handbuch der Kinderkrankheiten. III. 2. Tübingen 1878.

Zuber, Adénopathio trachéo-bronchique in Grancher-Comby-Marfan. Traité des maladıes de l'enfance. 

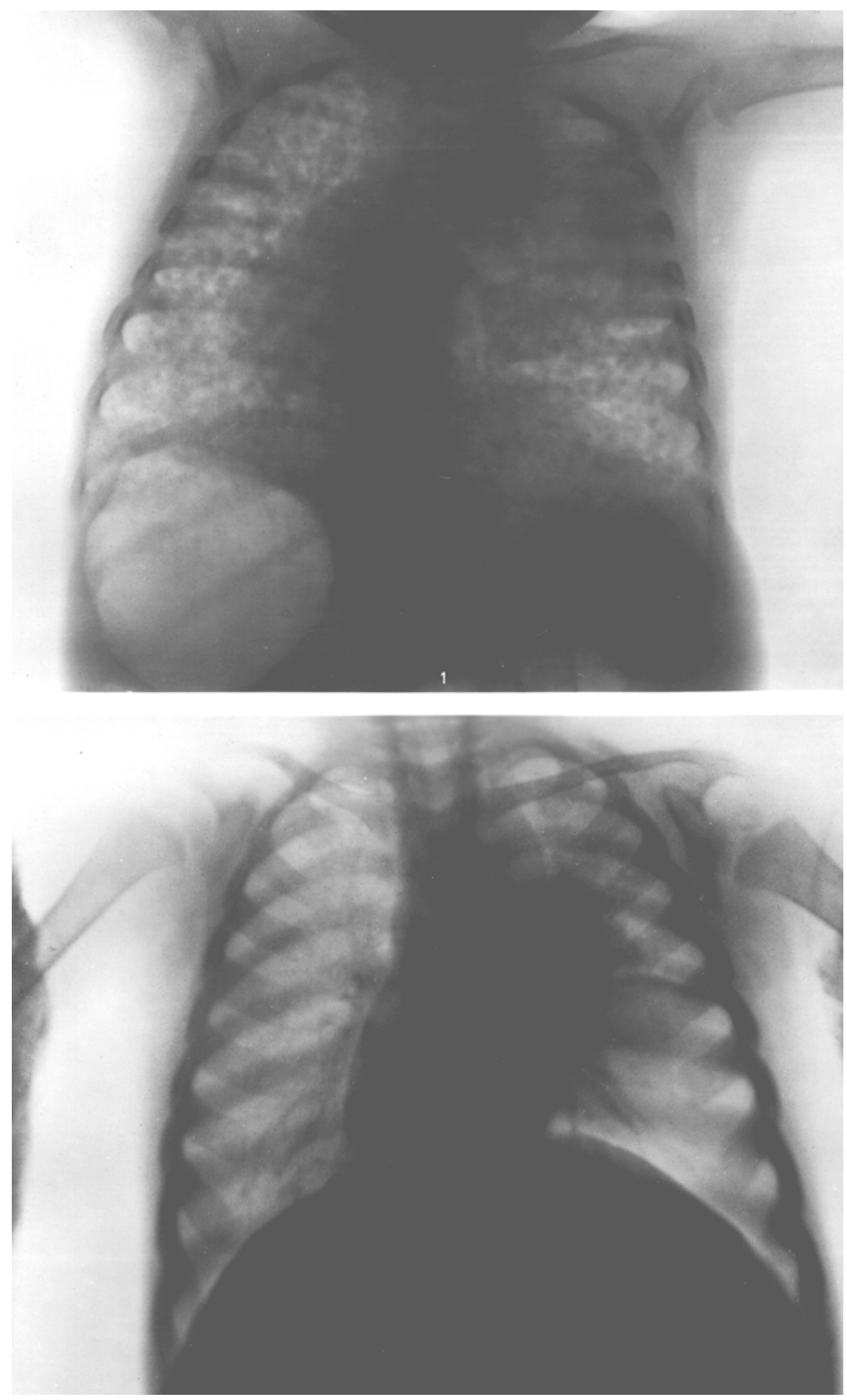